\title{
Anabases
}

ANABASES Traditions et réceptions de l'Antiquité

$8 \mid 2008$

Varia

\section{La référence à l'Antiquité dans la philosophie morale de Hume}

\section{Philippe Saltel}

\section{(2) OpenEdition}

1 Journals

Édition électronique

URL : http://journals.openedition.org/anabases/126

DOI : 10.4000/anabases. 126

ISSN : 2256-9421

Éditeur

E.R.A.S.M.E.

\section{Édition imprimée}

Date de publication : 1 octobre 2008

Pagination : 67-82

ISSN : 1774-4296

\section{Référence électronique}

Philippe Saltel, «La référence à l'Antiquité dans la philosophie morale de Hume », Anabases [En ligne], 8 | 2008, mis en ligne le 01 juillet 2011, consulté le 20 octobre 2019. URL : http:// journals.openedition.org/anabases/126; DOI : 10.4000/anabases.126 
Anabases 8 (2008), p. 67-82.

\section{La référence à l'Antiquité dans la philosophie morale de Hume}

PhilipPe SALTEL

$\mathrm{H}$

UME NOUS A LAISSÉ DEUX VERSIONS DE SA MORALE systématiquement exposée : le Livre III du Traité de la nature humaine (1740) et l'Enquête sur les principes de la morale (1751) ; or les deux ouvrages présentent des différences d'un autre genre que celles qui séparent les deux versions de sa philosophie de la connaissance, le Livre I du Traité (1739) et l'Enquête sur l'entendement humain (1748). Sur la question de la connaissance, le cour de la doctrine ne varie guère d'un texte à l'autre ; les ajouts ou suppressions de chapitres ont rapport à ce que l'on peut nommer " les retombées de l'analyse ", les conséquences de la théorie des impressions et des idées ou celles de la critique de la causalité. Certes, le style de l'Enquête est mieux travaillé, plus élégant, plus concis, mais si de telles différences formelles sont importantes pour le spécialiste, elles ne modifient qu'à la marge le message philosophique de l'Écossais.

Il en va autrement dans le domaine moral. En premier lieu, force est de constater que la doctrine a subi un infléchissement important : si Hume continue à suivre l'approbation et le blâme comme indices des vertus et vices qu'il entend "mettre en système ", l'explication psychologique des phénomènes d'approbation comme de ceux de la "propension vertueuse " qui vient concurrencer l'intérêt personnel ne dépend plus, dans l'Enquête, d'un mécanisme originellement amoral, la sympathie, mais bien d'un "sentiment " commun à toute l'humanité, et déjà moral, la bienveillance. À cette modification doctrinale majeure, qui rend la théorie de l'Enquête moins originale que celle du Traité, s'ajoute celle de la présentation du système empirique des vertus, le texte de 1751 faisant prévaloir l'organisation en quatre espèces de qualités louables, selon qu'elles sont utiles ou agréables, à la personne qui les possède ou aux autres personnes. Bien entendu, nous trouvons dans cette Enquête un style beaucoup plus soigné qui lui 
a fait attribuer par l'auteur le titre de "meilleur de tous [ses] écrits ${ }^{1}$ ». Mais, sur ce même plan, nous voudrions remarquer et étudier ici une autre caractéristique manifeste. L'Enquête, en effet, est démesurément beaucoup plus riche que le grand texte de jeunesse en anecdotes, références à l'histoire, citations d'auteurs, et particulièrement celles qui ont trait au monde ancien. Pour en donner une idée, le Traité ne cite que quelques noms d'empereurs ou rois de l'Antiquité ; la seconde version de la morale rapporte de nombreux passages des poètes, historiens, moralistes, philosophes grecs et romains, évoque la vie de plusieurs généraux, princes, héros et héroïnes, compare enfin en maints endroits les mœurs des anciens et celles des modernes. L'écriture humienne présente alors, sur ce point notamment, la tournure qui est celle des Essais moraux et politiques, ce recueil que Hume a déjà fait paraître en deux volumes (27 essais en tout), puis en un seul (de 26 essais, après un petit appendice de trois essais), avant de remanier à plusieurs reprises l'ensemble, puis d'y joindre en 1753 les deux Enquêtes (ainsi que, dans l'édition de 1758, la Dissertation sur les passions et l'Histoire naturelle de la religion) pour composer les Essais et Traités sur plusieurs sujets, c'est à dire la version de sa philosophie au profit de laquelle il répudie finalement le Traité de la nature humaine.

Nous voudrions nous interroger ici sur le sens de la référence à l'Antiquité dans le corpus des œuvres morales du philosophe. Dans la mesure où elle n'est pas égale, mais surtout seconde, cette référence doit bien répondre à une intention nouvelle de sa part, que nous tenterons de déterminer : il se pourrait qu'il ne s'agisse que de gagner un nouveau public, moins académique mais plus lettré, à sa proposition théorique ; il se pourrait aussi que cette référence ne soit pas seulement un procédé parmi d'autres, que l'antique réponde à d'autres exigences que le " goût du jour » : mais lesquelles ? Et puis, si l'on veut bien admettre qu'un tel changement dans " la manière " d'une philosophie morale résulte d'un geste philosophique qui n'est pas anodin, nous devons étudier les effets d'un tel geste: quelles sont les conséquences, pour une morale " de la nature humaine ", d'aller chercher ses preuves si loin de nous, c'est-à-dire loin de la modernité marchande et de la logique des intérêts ? En quelques mots qui simplifient à l'extrême mais restent fidèles à une distinction de Hume, quels infléchissements quant à « la matière " implique cet infléchissement de " la manière " ?

Les quelques remarques qui suivent procèdent de l'étude d'un petit texte placé en appendice de l'Enquête sur les principes de la morale, texte intitulé "Un dialogue ${ }^{2}$ ". Nous le proposons donc à une réflexion préalable, car il offre, selon nous, un véritable index de la référence à l'Antiquité dans les textes postérieurs au Traité de la nature humaine: voici donc un dialogue "en prologue» de l'examen des deux questions Paris, Presses universitaires de France, 2001, p. 95. 
susdites, celle des intentions et celle des conséquences de la référence aux Anciens chez ce grand Moderne qu'était David Hume.

\section{Prologue}

Le texte que nous proposons à l'étude se trouve donc à la fin de l'Enquête sur les principes de la morale; il vient après neuf sections de "recherches" (ce terme traduisant mieux celui d'Enquiries) particulièrement riches en exemples donnés par la littérature, l'histoire, ou " construits" par Hume lui-même. Suivent quatre appendices qui sont autant de dissertations plus serrées sur les éléments principaux de la doctrine, en tant qu'ils se prêtent à objections (le sentiment moral, la conception humienne de l'amour de soi, la vertu artificielle de justice, la distinction, contestée par Hume, entre volontaire et involontaire). Le lecteur trouve alors un dernier chapitre, présenté sous la forme d'un récit de dialogue, forme traditionnelle d'écriture philosophique que Hume donnera plus tard au traitement de la question de la religion des philosophes, c'est-àdire de la religion naturelle. De la sorte, notons-le, le philosophe aura montré dans la seconde Enquête trois manières d'écriture philosophique, celle de l'essai (la première Enquête a d'abord eu pour titre celui d'Essais philosophiques sur l'entendement humain), celle de la dissertation, celle du dialogue.

De quoi s'agit-il dans ce dialogue ? Le narrateur y relate une discussion avec son " ami Palamède ", homme lettré et grand voyageur (d'où le choix de ce prénom), au cours de laquelle Palamède raconte son séjour au pays appelé "Fourli ", un pays aux mœurs très éloignées, pour ne pas dire opposées, à celles de l'Europe moderne. Deux fictions sont donc emboîtées, celle du dialogue lui-même, celle du récit de voyage au pays imaginaire de Fourli : une discussion qui n'a jamais eu lieu constitue la mise en scène qui permet à l'auteur de conduire son lecteur vers un pays qui n'existe pas. À lire la relation ethnographique de Palamède, nous découvrons alors qu'elle sert de support à l'énumération de pratiques exotiques, mieux, à la description d'un système de pratiques et donc de valeurs qui, pour être complètement étrangères, n'en constituent pas moins la structure morale de cette étrange société, comparable au système linguistique qu'après tout elle accompagne.

Quand j'arrivai parmi eux, je découvris qu'il me fallait faire face à une double difficulté : d'abord celle d'apprendre les significations des termes dans leur langage, et puis celle de connaitre la valeur de ces termes, la louange ou le blâme qui leur étaient associés ${ }^{3}$.

Or les aventures de Palamède lui révèlent peu à peu les mœurs étonnantes des "Fourliens » : ils interprètent les éloges comme des affronts, ils éprouvent des amours homosexuelles, lesquelles ne choquent aucunement leurs épouses légitimes, ils approu-

Enquête..., p. 251. 
vent le meurtre et le parricide pour des motifs d'utilité personnelle, peuvent pousser l'ingratitude jusqu'au complot dans l'assassinat, endurent les effets de la colère d'autrui, considèrent le suicide de l'homme vieillissant, malade ou malheureux, comme le couronnement d'une vie, invitent les plus pauvres à partager leur maigre repas avec les plus riches. Un tel récit provoque alors l'emportement du narrateur, qui croit à une moquerie, puisque de telles mœurs

[...] dépassent tout ce que nous avons jamais lu au sujet des Mingréliens et des Topinambous ${ }^{4}$.

La colère du narrateur prépare le " coup de théâtre » du dialogue, la révélation, par Palamède, qu'il a présenté sous les traits de cette peuplade imaginaire les Athéniens, d'ailleurs très largement considérés :

Les amours des Grecs, leurs mariages et les dangers qu'ils faisaient courir à leurs enfants ne peuvent que vous frapper immédiatement. La mort d'Usbek [tué dans un complot organisé par son ami ingrat] est un pendant exact de celle de César ${ }^{5}$.

Hume a donc incontestablement cherché à dramatiser une objection que l'on pouvait faire à sa morale. En effet, l'une des caractéristiques de sa pratique philosophique est, comme tout lecteur peut s'en apercevoir, le soin qu'il apporte à opposer à ses hypothèses tout ce qu'il peut leur opposer, afin de lever ces difficultés, ce qu'il parvient à accomplir avec plus ou moins de réussite. Toutefois cette manière de faire appartient généralement à un discours argumentaire très sérieux et n’emprunte pas, d'ordinaire, la voie de la fiction ou le tour "léger " du récit imaginaire, qui incitent à rapprocher ce texte de certains passages de certains essais, dont quelques essais retirés pour leur trop grande légèreté, et qui nous portent à nous interroger sur les intentions du philosophe quand il évoque les Anciens, c'est-à-dire sur le sens qu'il donne à cette référence.

\section{Pourquoi Hume introduit-il l'exemple des Anciens dans sa théorie morale?}

La comparaison des deux versions de la morale humienne et, plus largement, du Traité et des textes postérieurs rend l'hypothèse d'un changement de stratégie très probable: le philosophe aurait cherché à atteindre un public élargi en satisfaisant notamment son goût. Or ce goût, et tout particulièrement dans l'Écosse de cette seconde moitié du XVIII , a mis l'Antiquité à la mode ; Hume, qui a lu très tôt les

4 Enquête..., p. 255-256. La Mingrélie se trouve au sud-ouest du Caucase, les "Topinambous » sont les indiens Tupinambas (Brésil) : voir les notes de l'édition française citée, p. 310, n. 3.

5 Enquête..., p. 256. 
auteurs latins, qui a appris le grec au début des années 1740, a les moyens de chercher à plaire par ce biais. Mais ce n'est pas tout d'avoir de tels moyens : encore faut-il que cela réponde à une exigence légitime, c'est-à-dire philosophique, quand bien même il s'agit encore de rhétorique. Il est donc nécessaire, sur ce point, de chercher plus loin. D'abord, parce que l'insuccès du Traité que Hume place à l'origine de son changement de style est tout relatif : pour un ouvrage anonyme, le Traité a connu, en vérité, une certaine publicité, comme l'attestent les recensions parues à l'époque ${ }^{6}$; ensuite parce que les critiques adressées à l'ouvrage portent notamment sur sa difficulté, due à trop d'abstraction, ce qui en limite la portée; enfin et surtout parce qu'elles touchent une distinction déjà faite par Hume dans le Traité, et qui ne sera jamais reniée par lui, entre deux manières de philosopher, qui ont toutes deux leurs avantages et leurs inconvénients, celle des salons, clubs et autres lieux de conversation (la "philosophie facile") et celle qui se pratique dans la solitude des cabinets d'étude (la "philosophie sérieuse ») : signalons au moins une occurrence majeure, à la fin du livre III du Traité, dans laquelle il oppose, sur la question de la morale, l'anatomiste (qu'il a été) et le peintre (Hutcheson est particulièrement visé) ${ }^{7}$. Or les écrits postérieurs, qui reprennent cette dichotomie, témoignent d'une évolution du philosophe sur ceci qu'il ne juge plus incompatibles les deux façons de faire et pense pouvoir dépasser les inconvénients de l'une et de l'autre par la recherche d'une voie moyenne : plutôt, donc, que d'un tournant imposé par l'échec, nous devrions parler d'un approfondissement de la réflexion sur les conditions du travail philosophique en ces temps où la pratique philosophique est disséminée, le recours à l'expérience, généralisé, et où sont apparues de nouvelles conditions d'existence. Il faudrait peindre, autant que dessiner : mais pourquoi donc?

L'exemple de notre texte nous éclaire sur ce point : la mise en scène de la référence aux Anciens permet en effet le triomphe final du narrateur, qui propose une analyse de chacune des coutumes des Grecs ou des Romains, une analyse destinée à les dériver des principes exposés dans le corps de l'ouvrage, les catégories d'utilité ou d'agrément, pour autrui ou pour soi-même. Hume va donc chercher si loin une confirmation de ses hypothèses, une confirmation on ne peut plus expérimentale qui se plie au principe même de l'enquête : il s'agit bien de faire le relevé de ce qui est approuvé pour en dresser le catalogue raisonné ; quelques mœurs anciennes paraissent-elles faire exception ? le philosophe montre que ce n'est qu'une apparence :

Il apparaitt qu'il n'y a jamais eu de qualité recommandée par quiconque comme vertu ou comme perfection morale, si ce n'est en raison de sa nature utile ou agréable à son possesseur lui-même ou aux autres. [...]Toutes les différences en morale peuvent donc être

6 Voir E.-C. MOSSNER, “The Continental Reception of Hume's Treatise, 1739-1741", Mind 56 (1947), p. 31-43.

7 Traité de la nature humaine, III : La Morale, 3.6., trad. franç. par Ph. SALTEL, Paris, Flammarion, 1993, p. 248-251. 
ramenées à ce fondement général et elles peuvent être expliquées par les divers angles sous lesquels les gens envisagent ces circonstances ${ }^{8}$.

On voit donc quelle fonction est assignée à la référence aux mœurs anciennes : une fois dramatiquement présentées comme les plus éloignées des nôtres, voire " diamétralement opposées " comme Hume le fait dire à Palamède, elles peuvent faire l'objet d'une tentative de réduction aux principes généraux de la morale, tentative déterminante si l'on veut les élever au rang de "principes de la nature humaine ». En quelques mots, c'est dans l'Antiquité qu'une morale "empirique " trouve son experimentum crucis. Notre texte le manifeste clairement, dans la mesure où il se conclut par la disqualification des mœurs extravagantes, celles d'un Diogène dans les temps anciens, quand la philosophie avait de l'influence sur la conduite, celles d'un Pascal dans les temps modernes, quand la religion a pris cette place : non sans humour, le philosophe écossais repousse cette dernière objection possible d'un revers de la main :

Une expérience [...] qui réussit dans l'air ne réussit pas toujours dans le vide 9 .

Cela signifie pour nous qu'une fois ces extravagances rejetées dans les marges de l'enthousiasme ou de la superstition, il n'est pas de mœurs réelles et de morale concrète plus éloignées des nôtres que celles des Anciens, raison pour laquelle il n'est pas de confirmation sérieuse d'une proposition en philosophie morale qui puisse se dispenser de ce test.

Il vaut enfin la peine de considérer dans quels termes cette confirmation se fait, comment Hume entend réduire la différence des Anciens et des Modernes, car cela, nous semble-t-il, est susceptible de nous mettre dans la voie d'une compréhension de l'évolution de sa philosophie et de sa contribution à celle de la philosophie elle-même. Il dit en effet, dans la réponse du narrateur à l'objection de Palamède, sur quels points, selon quels « angles " les morales peuvent diverger sans pour autant qu'il soit de la sorte porté atteinte aux "principes généraux ». Cela se fait en deux temps.

[1] Quelquefois les hommes different dans leur jugement sur l'utilité d'une coutume ou d'une action, quelquefois aussi les circonstances particulières des choses rendent une qualité morale plus utile que les autres et lui ajoutent une préférence particulière ${ }^{10}$.

Suivent alors trois cas de préférences ainsi déterminées par le jugement ou les circonstances: la définition du courage, selon qu'on est en temps de guerre ou en temps de paix, la plus grande convenance, à certaines époques, des qualités de magnanimité, grandeur de vues, dédain de l'esclavage, rigueur inflexible - nous pouvons rassembler ces vertus sous le nom de "grandeur d'âme " ou de "générosité ", au sens classique du terme -, enfin le rapport au luxe, lequel peut être " ruineux ou pernicieux pour un 
homme né en Suisse ${ }^{11}$ " mais non pour un Français ou un Anglais. Le principe de l'utilité, d'autant plus important que nous savons quels développements ultérieurs la philosophie britannique lui donnera, ne serait pas remis en cause par de telles variations ; toutefois, nous ne pouvons pas ne pas nous interroger sur la tension ainsi induite entre ce qui est général et ce qui est variable, dans la mesure où la valeur de certaines qualités, courage, grandeur d'âme, raffinement, pourrait aller de " presque tout " le mérite personnel à " presque rien " dans ce mérite, selon les situations. Il serait alors difficile de passer de l'affirmation de l'utilité comme principe à la description de ce qui est utile.

Le second mode de variation est alors identiquement exposé : Hume présente un facteur de diversité des mœurs qui n'affecterait pas le système d'ensemble puis trois cas, encore, qui en illustrent l'opération.

[2] Les coutumes différentes ont aussi une certaine influence, tout comme les utilités différentes, et, en donnant à l'esprit un premier penchant, elles peuvent produire une propension plus importante, soit aux qualités utiles, soit aux qualités agréables, à celles qui concernent le moi ou à celles qui s'étendent à la société ${ }^{12}$.

L'effet des coutumes est exemplaire, selon Hume, dans le cas des relations entre les deux sexes, celui de la valeur des qualités sociales agréables, l'art de la conversation, l'humour, la politesse, etc., enfin celui d'un ensemble de variations qui relèvent de la situation générale d'une nation, son type de gouvernement, sa richesse, l'état de son savoir : toutes choses qui suscitent des habitudes comportementales distinctes dont le philosophe modère aussitôt l'influence (et celle de toutes les différences énumérées dans ce second volet) en concluant que cela n'agit vraiment que sur les jeunes gens, portés à la recherche de l'agrément et du succès, quand les hommes mûrs préferent des qualités plus solides. Cela est conforme à sa philosophie qui attribue un effet d'uniformisation à l'expérience acquise par les voyages, les rencontres, les discussions et les lectures, de sorte que le dépassement des sympathies premières, le raffinement des passions, l'accès à la vertu résultent, selon cette philosophie, de l'exercice de la vie et s'acquièrent plus qu'ils ne s'imposent.

En dépit de telles remarques qui limitent l'effet des variations prises en considération, Hume vient ainsi d'introduire dans ses recherches morales un élément complémentaire qui n'est pas anodin, car l'application des principes à des sociétés aussi différentes que celles des Anciens et celles des Modernes suppose désormais l'ajout d'explications correctrices susceptibles de rendre compte de la façon dont la nature humaine adapte, pour ainsi dire, ses tendances les plus générales à des situations qui la font varier elle-même. Dès lors la méthode ascendante, qui va des qualités effectivement louées ou blâmées aux principes généraux de louange et de blâme doit être sur les sciences et les arts a été publié en 1750 . 
complétée par une démarche descendante, qui explique quelles valeurs sont privilégiées dans quelles situations historiques, et surtout lesquelles correspondent aux principes dans la situation contemporaine de l'écriture humienne, la société européenne marchande, éclairée, sécularisée, constituée en nations soumises aux impératifs de leur cohésion et de la liberté de leurs citoyens. Il importe ici de remarquer, pour finir cette première partie de notre étude, que la liste de valeurs susceptibles de varier correspond à celles qui sont principalement étudiées dans les Essays, Moral and Political dont Hume a publié, en 1748, une édition importante (26 essais), avant l'ajout des Political Discourses (1752) et la réunion avec les deux Enquiries (1753). En somme, l'évocation des mœurs des anciens aura non seulement répondu à l'intention de gagner un lectorat élargi, grâce à un style réformé pour lequel cette référence joue un rôle important, mais aussi à celle de confirmer par une sorte d' " expérience cruciale » la théorie morale, et enfin à celle de renvoyer au programme des Essais et ainsi de consolider le lien entre les deux parties, les deux manières de sa philosophie, voire, comme nous le pensons, les deux registres de la pensée morale.

\section{Mœurs anciennes et morale moderne}

Il y a donc, selon notre philosophe, un certain nombre de questions « secondaires » au sujet desquelles la variation peut être grande entre telles et telles mœurs, voire la plus grande concevable, entre mœurs modernes et mœurs anciennes, sans que, pour autant, les principes ne puissent varier. Néanmoins, parce qu'elle est extrême, la comparaison des sociétés modernes et des sociétés anciennes peut nous paraitre affecter la rigueur du principe général ; elle offre en outre à la conscience moderne un contrepoint susceptible de mettre en évidence ses "préjugés " et d'éclairer sa situation, de sorte que l'espace d'une anthropologie comparée se trouve ainsi ouvert et vienne en complément, voire en amendement de la psychologie morale, c'est-à-dire d'un chapitre fondamental de la fameuse «science de la nature humaine ".

Nous devons tout d'abord constater que l'élargissement du champ expérimental est contemporain d'ajustements théoriques significatifs pour lequel il fournit des données nouvelles. Il en est ainsi, notamment, des passions égoïstes. Tout lecteur de Hume sait que la seconde version de sa morale, dans l'Enquête, est orientée vers une contestation des théories de l'intérêt et de l'amour propre, qu'elles soient signées de Hobbes, La Rochefoucauld ou Mandeville, une contestation très sérieusement argumentée dans l'Appendice II : Hume y présente en effet l'idée nouvelle que l'égoïsme pourrait n'être qu'une passion seconde, après tout sans autre objet que celui que lui donne une propension première, quelle qu'elle soit :

S'il n'y avait aucun appétit d'aucune sorte qui précède l'amour de soi, cette propension égoïste n'aurait presque jamais l'occasion de s'exercer car, dans un tel cas, nous aurions eu 
à éprouver des peines et des plaisirs à la fois rares et faibles, et n'aurions que peu de bonheur et peu de malheur à rechercher ou à éviter ${ }^{13}$.

Il convient, à ce sujet, de remarquer que l'un des arguments en faveur d'une telle réorganisation du tableau des passions consiste dans l'observation que des passions méchantes peuvent aller aussi loin dans l'oubli de soi que des passions bienveillantes, qu'il y a une sorte de "générosité " chez le méchant, comme l'avait déjà noté Virgile que Hume, en cet endroit, paraphrase.

Qui ne voit que la vengeance, par la seule force de la passion, peut être si avidement poursuivie qu'elle nous fait négliger toute considération de bien-être, d'intérêt et de sécurité et, comme pour certains animaux rancuniers, nous pousse à infuser nos âmes dans les blessures que nous infligeons à autrui ${ }^{14}$ ?

L'analyse des passions égoïstes s'enrichit, croyons-nous, dans les écrits postérieurs au Traité qui l'enserrait dans la doctrine d'une partialité primitive (certes dépassée par une extension naturelle ou artificielle) et négligeait de ce fait leur articulation aux autres passions. Une place singulière leur est désormais attribuée. Ainsi, dans l'essai De la dignité ou de la bassesse de la nature humaine (1748), la vanité est présentée comme douée de cette même plasticité :

Il en va en effet différemment de la vanité et des autres passions. [...] la passion pour la gloire varie en se pliant toujours au goût et au tempérament particuliers de ceux qui en sont animés. La même vanité qui poussait Néron à conduire un char poussait Trajan à gouverner l'empire de manière juste et habile. Aimer la gloire que procurent les passions vertueuses, c'est prouver que l'on aime la vertu elle-même ${ }^{15}$.

Rien, à vrai dire, ne nous permet de conclure qu'une telle modification soit l'effet direct de la référence nouvellement multipliée aux auteurs et acteurs de l'Antiquité grecque ou romaine ; tout au moins cette référence fournit-elle des exemples, voire des arguments d'autorité, à une pensée qui développe, dans sa seconde période, une psychologie morale indépendante des préjugés modernes, et notamment de celui qui voudrait que seules les qualités volontairement acquises, et non pas les aptitudes naturelles, puissent recevoir le nom de vertu. Ce préjugé, le Traité l'attribue aux intentions générales de ceux qui veulent régler les mœurs, tandis que l'Enquête, plus directe, rend l'influence de la théologie sur la morale responsable d'une telle déformation. Hume a toujours voulu délivrer la morale de la tutelle religieuse, et l'opposition des morales de

13 Enquête..., App. II, p. 225.

14 Enquête..., p. 225 : la fin de la phrase traduit quasiment un passage des Géorgiques: Animasque in vulnere ponunt (IV, v. 238), que Hume cite en note.

15 Of the Dignity and Meanness of Human Nature, trad. franç. De la dignité ou de la bassesse de la nature humaine, in Essais..., p. 225. 
l'Antiquité aux préjugés du temps a toujours servi ce but chez lui ; néanmoins, elle en sert évidemment d'autres, parmi lesquels le raffinement de la théorie.

Nous en trouvons un exemple dans le cas du courage. Cette vertu, le Traité la place en équivalent masculin des vertus de chasteté et de pudeur chez les femmes, c'està-dire en "point d'honneur " qui «tire en grande partie son mérite de l'artifice ${ }^{16}$ ", bien qu'il soit, comme toutes les vertus " de grandeur ", directement approuvé pour son agrément (tandis que l'humilité, soutenue par la religion chrétienne, est désapprouvée comme bassesse au-delà des seules apparences convenant à la vie sociale ${ }^{17}$ ). Or l'essai sur « Les caractères nationaux " raffine le traitement de cette question.

On observe qu'en général, de toutes les qualités d'une nation, le courage est la plus incertaine, car elle n'est exercée que par intervalles et par un petit nombre d'hommes. [...] Si l'on veut entretenir le courage, il faut le faire par la discipline, l'exemple et l'opinion ${ }^{18}$.

Suit une "preuve " que le courage dépend de l'opinion, à savoir que les Doriens ont toujours été jugés plus courageux que les Ioniens, à l'exception des Athéniens : ce n'est donc pas la nature qui produit ici ou là des peuplades courageuses, mais bien l'artifice qui met en place les moyens d'une valorisation et, par suite d'une promotion de cette vertu dans une population. Il n'y a rien là qui aille beaucoup plus loin que la règle énoncée dans le Traité; mais "l'incertitude" du courage est introduite ainsi. Cette notion se trouve alors reprise dans un autre essai, Du raffinement dans les arts (1752), où un passage traite de l'effet prétendu d'amollissement des hommes par le raffinement de leur nation. Voici ce qu'écrit Hume :

Il n'y a pas lieu de craindre que les hommes, en perdant cette férocité, se dessaisissent aussi de leur esprit martial, ni qu'ils deviennent moins intrépides et moins vaillants à défendre leur patrie ou leur liberté. [...] Et si la colère, cet aiguisoir du courage, perd quelque chose de son aspérité sous l'effet de la politesse et du raffinement, le sens de l'honneur, principe plus fort, plus constant et plus aisé à gouverner, gagne une nouvelle vigueur grâce à l'élévation du génie qui naît de la connaissance et de l'éducation ${ }^{19}$.

On voit donc dans cet essai, qui avait pour titre primitif $O f$ Luxury, que le rapport aux biens extérieurs, précisément aux biens luxueux, produit selon Hume une modification importante dans l'équilibre général des passions et leur contribution à la production d'une vertu artificielle : dans la société moderne, l'honneur sera le ressort de ce qui pouvait s'appuyer autrefois sur la colère. Alors il faut bien reconnaître que c'est la même chose et ce n'est pas la même chose d'être courageux ici ou là, non pas seulement d'après l'effort que fournit une cité pour cultiver en son sein une classe d'hommes 
courageux (Athènes en est l'exemple), mais aussi d'après les conditions qui donnent à cet effort son efficacité propre.

Il ressort de ces deux exemples qu'une théorie morale n'est désormais plus possible sans l'accompagnement de l'étude des variables qui fournissent à ses principes leurs modalités propres. Dans ces conditions, Hume va jusqu’à écrire, dans le même essai sur la question classique du luxe, que

[...] pas plus ici que dans les autres questions morales, la frontière entre le vice et la vertu ne peut être fixée exactement ${ }^{20}$.

Pourtant, nous constatons bien un effort vers plus d'exactitude dans la recherche de cette "frontière ", effort sans lequel, il est vrai, Hume ne serait pas philosophe, ne serait pas le "sceptique mitigé ", " académique ", qu’il prétend. Cet effort se déploie en deux sens : d'une part, il porte sur l'élucidation des moments de l'histoire des valeurs morales et des causes qui les font varier; d'autre part, il porte sur le moment présent, sur la pensée de ce moment, c'est-à-dire aussi bien sur la "critique » des valeurs du temps, qui en interroge déjà l'avenir.

La thématique de l'histoire des mœurs prend, à l'époque de Hume, un tour particulier qui est celui de la question des caractères nationaux déjà évoquée. Discutant les thèses de John Arbuthnot ${ }^{21}$ et donc, par avance, celles de Montesquieu qu'il ne connaît pas encore, Hume fait prévaloir les " causes morales " sur les " causes physiques » dans la détermination des mœurs particulières de telle ou telle nation. Il importe de remarquer, eu égard à notre sujet, l'importance considérable de la référence aux cités antiques dans l'essai Of National Characters. Puisqu'elles constituent en quelque sorte des nations, les cités de l'Antiquité valent parmi tant d'exemples des quelques causes morales d'un caractère national, par exemple de ceci que les mœurs dépendent grandement de la sympathie qui fait perdurer entre concitoyens un type déterminé de disposition: ainsi s'explique qu'en dépit du voisinage, Athéniens et Thébains soient si différents, beaucoup plus que les habitants d'Athènes et ceux du Pirée, n’en déplaise à Plutarque ${ }^{22}$; ainsi s'explique l'influence des changements historiques, modifications dans le gouvernement, mélanges à d'autres peuples, etc. : Grecs anciens et Grecs modernes en donnent l'exemple (ingéniosité, industrie et vivacité d'un côté, stupidité et indolence de l'autre), de même Romains anciens et Romains modernes (candeur, amour de la liberté et bravoure contre fausseté, lâcheté et servilité) ; à l'inverse la civilité des Français s'oppose à la barbarie des Gaulois ${ }^{23}$. Mais en même temps, les nations

Essais..., p. 442.

An Essay concerning the Effects of Air on Human Bodies (London, 1733).

Hume interprète probablement un passage des Vies parallèles consacré à Thémistocle : il compare, non sans malice, Saint-James et le quartier des docks à cette opposition prétendue entre le climat d'Athènes et celui du Pirée.

Sur tout cela, voir Des caractères nationaux, Essais..., p. 415-416. 
anciennes ne sont pas n'importe quelles nations : elles sont premières et toute l'histoire en dérive. Sur ce plan, elles représentent l'heureuse distribution d'une nature aveugle, où commence véritablement l'histoire :

Mais bien que la nature produise en grande abondance les esprits et tempéraments les plus divers, il ne s'ensuit pas qu'elle les produise toujours dans les mêmes proportions, ni qu'elle mélange de la même manière dans chaque société ces ingrédients que sont l'industrie et l'indolence, le courage et la lâcheté, l'humanité et la brutalité, la sagesse et la folie. Si dans l'enfance d'une société l'une de ces dispositions se trouve en plus grande abondance que les autres, elle continuera naturellement de prévaloir dans le composé et donnera une teinte particulière au caractère de cette nation ${ }^{24}$.

L'Antiquité a reçu en héritage naturel une palette de vertus digne de la plus grande estime, comme en témoigne un fragment de phrase de l'essai consacré à La populosité des nations antiques:

Certains admirateurs passionnés des anciens et partisans zélés de la liberté civile (ces deux sentiments étant dans l'ensemble extrêmement justes, ils sont presque inséparables) ${ }^{25}$...

L'histoire des mœurs peut donc être reconstituée sur une telle base, à l'aide des principes d'après lesquels l'action du gouvernement, la diffusion des langues et des religions, la situation de l'économie générale, l'inconstance des affaires humaines, etc., font varier le complexe originel. Par exemple, «Rome n'est plus dans Rome » et n'est plus elle-même non pas à cause du luxe, mais en raison d'erreurs politiques majeures :

Mais il serait aisé de prouver que ces écrivains se trompèrent sur les causes des désordres de la République romaine, et qu'ils imputèrent à tort au luxe et aux arts ce qui découlait en réalité d'un gouvernement mal réglé et de l'extension illimitée des conquêtes ${ }^{26}$.

Il y a donc chez Hume un premier essai d' "anthropologie pratique ", au sens large encore entendu par le Kant des Fondements d'une Métaphysique des mours; mais cette recherche ne saurait, chez lui, être séparée de celle des principes généraux que le philosophe de l'expérience entend extraire du témoignage humain; l'une et l'autre " enquêtes " sont enchevêtrées, sans doute d'une façon comparable à celle qui préside à la combinaison de plusieurs règles de jugement chez l'esthète, selon Hume : doué de délicatesse, aidé par sa pratique, l'expert esthétique sait comparer sans se laisser enfermer par le préjugé, mais en usant plutôt de son bon sens ${ }^{27}$. Et nous pouvons oser la

Essais..., p. 412.

Of the Populousness of Ancient Nations, trad. franç. De la populosité des nations antiques, Essais..., p. 559.

Du raffinement dans les arts, Essais..., p. 450-451.

Nous reprenons l'énumération des qualités de l'esthète, telle qu'elle est présentée dans l'essai Of the Standard of Taste, trad. franç. De la norme du goût, Essais..., p. 709. 
proposition que, pour ce philosophe qui compare fréquemment le jugement moral et le jugement de goût, une expertise philosophique, en matière morale, requiert cette large documentation de l'histoire des mœurs.

Elle la requiert d'autant plus que l'objet principal est bien la moralité de ce temps, comme en témoignent les textes, au premier rang desquels les Essays, destinés à des lecteurs contemporains du philosophe. Or pour s'adresser à cette époque dans laquelle les lettrés et le large public cultivé partagent une grande admiration de l'Antique, les mœurs, les goûts, les jugements des Anciens ne sont pas seulement reçus par Hume comme moyens pour sa philosophie ou comme éléments de son anthropologie historique, ils sont véritablement utilisés à des fins précises qui toutes visent, plus ou moins, l'amélioration de l'Europe moderne.

En premier lieu, les mondes anciens s'offrent à la comparaison avec le monde moderne, et à une comparaison qui n'a pas pour seule visée la détermination des variantes possibles du jugement moral, mais également l'estimation philosophique de la modernité. Ainsi Hume explique-t-il que la puissance militaire des cités antiques, très considérable si on la rapporte à la taille de leur population, dépend de l'absence de luxe et de commerce chez elles, comme le montre le cas extrême des Spartiates :

Il est évident que le travail des Ilotes n’aurait jamais pu pourvoir à la subsistance d'un si grand nombre de Spartiates si ces derniers avaient vécu dans l'aisance et la délicatesse $[\ldots]^{28}$.

Cela sert non pas tant à regretter le manque de puissance ou d'intégration martiale des nations modernes qu'à proposer une règle de compréhension de leur situation :

De même que l'ambition du souverain doit limiter le luxe des individus, de même le luxe des individus doit limiter la force du souverain et mettre un frein à son ambition ${ }^{29}$.

Dès lors, non seulement Hume s'oppose à ceux qui se demandent «si les souverains ne pourraient pas revenir aux maximes politiques de l'Antiquité 30 ", mais une telle observation explique pourquoi le seul régime constitutionnel ne peut être rendu responsable du degré de raffinement d'un peuple, les Français développant sous un prince absolu un commerce important, et par conséquent un grand raffinement... comparable à celui des Grecs, mais ici Hume ne songe plus à Sparte mais à Athènes.

Les Anglais sont peut-être de plus grands philosophes, les Italiens de meilleurs peintres et de meilleurs musiciens, mais les Français sont le seul peuple, à l'exception des Grecs, à

Of Commerce, trad. franç. Du commerce, Essais..., p. 430.

Essais..., p. 430.

Essais..., p. 432. 
avoir produit à la fois des philosophes, des poètes, des orateurs, des historiens, des peintres, des architectes, des sculpteurs et des musiciens ${ }^{31}$.

Toutefois le philosophe, passé maître dans l'art de la nuance, n'ignore pas quelles limites l'absolutisme impose au commerce, de sorte que se maintient en France un frein au raffinement :

Si donc le commerce a tendance à décliner sous les gouvernements absolus, ce n'est pas, selon moi, parce qu'il y est plus en danger, mais parce qu'il y est moins honoré ${ }^{32}$.

En établissant une "balance" entre puissance militaire et développement commercial, Hume assigne à l'Antiquité la fonction d'un modèle de compréhension de la modernité ; cela va même jusqu'à l'analogie, que le philosophe pense profitable à la proposition de saines conjectures sur l'avenir. Prenant acte de la sécularisation de l'Europe moderne, il juge en effet que nous sommes revenus, d'une certaine façon, dans les conditions du monde ancien, ce qui constitue selon nous la véritable raison pour laquelle il ne critique guère "l'Antiquité à la mode ${ }^{33}$ ", se plie à cette mode, allant même jusqu'à proposer des mises en scènes ou des épisodes mythologiques ${ }^{34}$ qui composent, chez lui, un registre secondaire d' "Antiquité imaginaire " destinée à cette Europe-là :

Aujourd'hui que l'humanité a enfin secoué ce joug [celui de l'Église de Rome], les choses sont presque revenues à leur situation initiale, et l'Europe actuelle est la copie sur une grande échelle de ce qu'était autrefois la Grèce en miniature ${ }^{35}$.

Utile point de comparaison, élément d'une analogie que Hume pense féconde puisque la diversité des nations, diversité de perspectives, ne peut qu'être profitable à

Of Civil Liberty, trad. franç. De la liberté civile, Essais... p. 231.

Essais..., p. 233.

33 Il y a toutefois un principe d'exception : Hume récuse tout usage rétrograde de la référence à l'Antiquité ; nous venons d'évoquer le cas de la proposition d'un retour aux régimes politiques de l'Antiquité ; un peu plus haut, nous avons incomplètement cité un passage de l'essai sur La populosité des nations antiques (n. 22) présentant l'amour conjoint de l'Antiquité et de la liberté civile, chez des admirateurs qui, selon la suite du texte, ne peuvent s'empêcher de regretter la perte de l'esclavage et « seraient bien aise de soumettre la plus grande partie du genre humain à une servitude et à une sujétion bien réelles " (Essais..., p. 559).

34 Il en est ainsi du développement fictionnel que Hume donne au régime temporairement matriarcal des Scythes, dans l'essai Of Love and Marriage puis de la légende d'associations formées par Jupiter entre Sagesse, Vertu et Confiance, d'une part, Folie, Vice et Défiance, d'autre part, compagnies malheureuses et vite "recomposées" aux dépens des hommes qui s'y trompent encore et croient à tort que la Confiance accompagne les plus nobles de ces Dames. Cet essai a été retiré en 1760. arts et des sciences, Essais..., p. 279. 
l'avancée du savoir par la critique ${ }^{36}$, l'Antiquité est enfin opposée par lui aux " préjugés " de la modernité pour en limiter l'influence. C'est évidemment le cas pour ce qui concerne la définition même de la vertu, au sujet de laquelle il y a lieu, il est temps, en quelque sorte, de contester une conception trop étroite, produite par l'emprise de la religion chrétienne sur les distinctions morales. Sans revenir sur ce point bien connu et que nous avons précédemment évoqué, nous pouvons maintenant le rapprocher de la compréhension humienne de l'époque et des conditions qu'elle offre à l'intelligence philosophique; c'est en effet parce que «l'humanité a secoué ce joug " qu'il est possible de chercher à construire la morale sur une base nouvelle, depuis un point de vue plus dégagé pour lequel les conceptions anciennes ne sont plus si éloignées mais font en quelque sorte "concurrence " aux morales plus récentes et aux croyances qu'elles ont pu produire dans la conscience européenne. En ce sens, dès le Traité, mais encore dans l'Enquête puis dans les Essais, Hume se place à l'avant-garde de ce "goût pour l'Antique » qui n'est qu'un effet de la sécularisation et, en quelque sorte, le retourne vers ses promoteurs pour en signifier toutes les exigences : les " admirateurs de l'Antiquité " sont ainsi conviés à entendre ce qu'elle a pu dire de la vertu et qui s'est trouvé, quelques siècles durant, placé sous le boisseau. L'essai sur «le suicide " que Hume a renoncé à publier de son vivant ${ }^{37}$ procède de ce même positionnement, à la fois conséquent (puisque Hume ne fait jamais que pousser à leurs aboutissants philosophiques les préférences de ses contemporains) et dangereux (puisqu'il se place en quelque sorte "en avant " de son temps en agissant ainsi).

\section{Conclusion}

Revenons au "Dialogue " qui nous a servi d'index pour un tour d'horizon général des différents usages de l'Antiquité dans l'écriture philosophique humienne. Cette référence montre principalement que les situations suscitent des jugements de valeur différents et produisent ainsi, en certaines circonstances, certaines organisations du tableau des vertus : ainsi, par exemple, le courage prend la première place en temps de guerre. Mais elles donnent aussi aux vertus des modalités distinctes : ce ne sont pas les mêmes passions qui soutiennent ce courage dans des conditions de vie rudes ou dans des sociétés raffinées. Enfin, les coutumes acquises par imitation expliquent le tour particulier de certaines façons d'exister : par exemple, en confinant leurs épouses au domicile et en

36 Ainsi la philosophie de Descartes, puis celle de Newton, ont connu, connaissent encore à l'époque de Hume des oppositions venues des autres nations d'Europe (ibid.).

37 Of Suicide, trad. franç. Du suicide, Essais..., p. 671-692. On trouvera, p. 671, le récit des circonstances complexes de la publication de cet essai, que les Français ont pu lire en traduction alors même que la parution du texte original avait été préalablement suspendue par Hume. 
réservant leur galanterie à des amours homosexuelles, les Grecs privilégient l'utile ${ }^{38}$; les mœurs des Français, fort différentes, privilégient l'agrément mais prennent le risque des " désordres de l'amour ${ }^{39}$ ", de sorte qu'on ne sait si la galanterie (entendue comme forme particulière de politesse) est le moyen moderne de la domination masculine ${ }^{40}$ ou si elle conduit à un commerce si "galant" (en l'autre sens de la notion) que les hommes s'y retrouvent en esclavage ${ }^{41}$.

Ces différents exemples permettent au moins d'établir que la théorie morale nécessite, selon Hume, son complément par une histoire de la morale, mais aussi que rien n'indique qu'une telle histoire ait une orientation déterminable, le philosophe se défiant notamment de « la disposition à blâmer le présent et à admirer le passé 42 ». Au fond, elle donnerait plutôt un contrepoids sceptique à la positivité de la théorie morale, fixée en ses quelques "principes généraux » dont les effets varient selon le temps et le lieu.

Et sans doute avons-nous là le meilleur qualificatif pour les usages humiens de la référence à l'Antiquité : ils sont sceptiques. Nous entendons par là que le doute sur la force de conviction de la théorie, indépendamment de ses moyens d'illustration selon le goût du jour, la recherche d'expériences de confirmation, la distribution d'objets d'étude pour un développement non systématique relèvent d'une démarche philosophique scrupuleuse, hésitante, mais rigoureuse aussi, comme en témoignent les questions que Hume se pose, qu'il pose aux temps modernes par cette écriture philosophique en mode "contrapunctique", entre philosophie et histoire, mondes modernes et mondes anciens, une manière de philosopher que nous pouvons penser nécessaire non seulement à toute évaluation des valeurs du présent mais également à la possibilité que la théorie ait des effets sur la pratique, deux intentions philosophiques qui ont des racines bien profondes et à vrai dire bien anciennes.

Philippe SALTEL
Maître de conférences HDR de Philosophie moderne
Université de Grenoble
3, rue Brocherie - 38000 Grenoble
philippe.saltel@upmf-grenoble.fr

38 De la naissance et du progrès des arts et des sciences, Essais..., p. 288-292.

39 Of Polygamy and Divorces, trad. franç. De la polygamie et des divorces, Essais..., p. 344.

40 C'est la thèse soutenue dans De la naissance et du progrès..., Essais..., p. 290.

41 C'est la thèse soutenue dans De la polygamie..., Essais..., p. 344.

42 De la populosité des nations antiques, Essais..., p. 636. 